\title{
Severe disruption and disorganization of dermal collagen fibrils in early striae gravidarum*
}

F. Wang (iD, ${ }^{1}$ K. Calderone, ${ }^{1}$ T.T. Do, ${ }^{1}$ N.R. Smith, ${ }^{1}$ Y.R. Helfrich, ${ }^{1}$ T.R.B. Johnson, ${ }^{2}$ S. Kang, ${ }^{1}$ J.J. Voorhees ${ }^{1}$ and G.J. Fisher ${ }^{1}$

${ }^{1}$ Department of Dermatology and ${ }^{2}$ Department of Obstetrics and Gynecology, University of Michigan Medical School, Ann Arbor, MI, U.S.A.

Linked Comment: Sambi and Watson. Br J Dermatol 2018; 178:590-591.

\section{Summary}

\section{Correspondence}

Frank Wang.

E-mail: frawang@med.umich.edu

Accepted for publication

6 August 2017

\section{Funding sources}

This study was supported, in part, by a Dermatology Foundation Research Fellowship (F.W.). The Dermatology Foundation had no role in the design and conduct of the study; collection, management, analysis and interpretation of the data; preparation, review or approval of the manuscript or the decision to submit the manuscript for publication.

\section{Conflicts of interest}

None declared.

*Plain language summary available online DOI $10.1111 /$ bjd. 15895
Background Striae gravidarum (SG), or stretch marks of pregnancy, begin as erythematous streaks and mature into hypopigmented atrophic bands.

Objectives In order to investigate molecular alterations that may promote atrophy of SG, we investigated dermal type I collagen fibrils, which provide human skin with support.

Methods We obtained skin samples of recently developed, erythematous abdominal SG from pregnant women. To examine the organization of collagen fibrils, second-harmonic generation imaging was performed using multiphoton microscopy. Immunostaining was used to determine protein expression and localization of type I procollagen, the precursor of type I collagen fibrils. Realtime polymerase chain reaction was used to determine gene expression levels.

Results In control (hip) and stretched normal-appearing perilesional abdominal skin, dermal collagen fibrils were organized as tightly packed, interwoven bundles. In SG, collagen bundles appeared markedly separated, especially in the mid-to-deep dermis. In the spaces separating these bundles, loosely packed wavy collagen fibrils lacking organization as bundles were present. These disorganized fibrils persisted into the postpartum period and failed to form densely packed bundles. Numerous large fibroblasts displaying type I procollagen expression were in close proximity to the disorganized fibrils, suggesting that the fibrils are newly synthesized. Supporting this possibility, immunostaining and gene expression of type I procollagen were increased throughout the dermis of SG.

Conclusions Early SG display marked separation of collagen bundles and emergence of disorganized collagen fibrils that fail to form bundles. These alterations may reflect ineffective repair of collagen bundles disrupted by intense skin stretching. Persistent disruption of the collagenous extracellular matrix likely promotes formation and atrophy of SG.

\section{What's already known about this topic?}

- Dermal collagen fibrils are organized as densely packed bundles that provide support to human skin.

- Little is known about alterations involving collagen fibrils in striae gravidarum (SG), also known as stretch marks of pregnancy.

\section{What does this study add?}

- In recently developed SG, collagen bundles become markedly separated, and disorganized collagen fibrils emerge and fail to form bundles. 


\section{What is the translational message?}

- These alterations of collagen fibril organization likely promote atrophy.

- Therapies promoting organization of collagen fibrils into densely packed bundles may improve SG.

'Stretch marks', or striae distensae, are associated with obesity, growth spurts, corticosteroid excess or pregnancy. Stretch marks of pregnancy are known as striae gravidarum (SG) and affect $50-90 \%$ of women. ${ }^{1}$ Typically, lesions affect the abdomen and breasts, and less frequently, the thighs and buttocks. Initially, lesions appear as erythematous, and sometimes oedematous, streaks. Over months to years, they mature into permanent, scar-like, hypopigmented linear bands displaying a crinkled shiny surface, laxity and atrophy.

Prevention and treatment of SG are difficult, in large part because the molecular pathogenesis of SG remains unclear, with various components of the dermal extracellular matrix (ECM) being implicated. ${ }^{2-4}$ The dermal ECM provides human skin with elasticity and strength. ${ }^{5}$ Elasticity is provided by elastic fibres, which form a mesh-like network composing $2-4 \%$ of the dry weight of the dermis. ${ }^{6,7}$ Elastic fibres consist of many proteins. In the mid-to-deep dermis in particular, these fibres consist mainly of elastin, a polymer of cross-linked tropoelastin proteins. ${ }^{6,7}$ Strength, resiliency and support of human skin are mainly provided by type I collagen fibrils, which compose $80-90 \%$ of the dry weight of the dermis. ${ }^{8-10}$ Fragmentation of type I collagen fibrils, as observed in aged or photoaged skin, compromises structural support. ${ }^{10}$ Type I collagen fibrils are synthesized in dermal cells (fibroblasts) as a soluble precursor, type I procollagen. After secretion into the extracellular space, type I procollagen is processed to form long type I collagen fibrils. ${ }^{10,11}$ Type I collagen fibrils are organized and stabilized by cross-links to form bundles providing structural support. ${ }^{9-11}$ These bundles are interwoven together, ${ }^{12}$ and are densely packed in the mid-to-deep (reticular) dermis, but are thinner and more loosely packed in the upper (papillary) dermis. Various ECM components, including proteoglycans, glycoproteins, glycosaminoglycans and other types of collagen, associate with type I collagen fibrils, aiding their organization into bundles. ${ }^{9,10,13-17}$

It is generally believed that pathogenic alterations in striae distensae, including SG, involve collagen fibrils, ${ }^{18-20}$ elastic fibres, ${ }^{4,18,21-23}$ and/or other dermal ECM components, such as fibrillin. ${ }^{24}$ However, how and to what extent these ECM constituents become deranged is not clear. Recently, we analysed dermal elastic fibres in newly developed, erythematous abdominal SG in pregnant women. ${ }^{4}$ We observed severe disruption of the elastic network, accompanied by the emergence of newly synthesized, disorganized tropoelastin-rich fibrils, which persist and fail to form normal-appearing elastic fibres. These changes may compromise skin elasticity, contributing to laxity of mature SG. Here, we expand on those findings, focusing on the structure and metabolism of type I collagen fibrils in SG lesions. Our study includes the use of multiphoton microscopy to perform second-harmonic generation (SHG) imaging, a relatively novel technique for examining fibrillar collagens (including type I collagen), without relying on exogenous labelling. Compared with confocal microscopy, or other traditional methods that require staining of thin tissue sections, SHG imaging is capable of high-resolution imaging of thick skin sections and allows for enhanced, three-dimensional interpretation of collagen organization. Our findings provide insight into the formation of SG and the development of atrophy, as observed in mature lesions.

\section{Materials and methods}

\section{Skin samples, immunostaining and gene expression}

The study was approved by the Institutional Review Board at the University of Michigan, and conducted in accordance with the Declaration of Helsinki principles. Skin biopsies were obtained from 2004 to 2010 and immunostaining, image analysis and gene expression assays were performed as previously described. ${ }^{4,25}$ The antibodies used for staining are listed in Table 1. For real-time polymerase chain reaction, the primers and probes for type I procollagen, ${ }^{26}$ transforming growth factor- $\beta 1$ and $-\beta 3,{ }^{27}$ connective tissue growth factor, ${ }^{28}$ matrix metalloproteinase- ${ }^{29}$ and an internal control $(36 \mathrm{~B} 4)^{28}$ have been described previously. Sequences of primers and probes for heat shock protein 47, prolyl-4-hydroxylase and matrix metalloproteinase- 3 are provided in Table 2.

\section{Second-harmonic generation and immunofluorescence imaging using multiphoton microscopy}

Multiphoton microscopy was used to perform both SHG and immunofluorescence imaging during separate scans. After fixation in 2\% paraformaldehyde for $20 \mathrm{~min}$ and protein blocking (Biogenex Laboratories, Fremont, CA, U.S.A.) for $30 \mathrm{~min}$, skin sections (up to $100 \mu \mathrm{m}$ ) were incubated with primary antibodies (Table 1). Following protein blocking for $20 \mathrm{~min}$, sections were incubated with antirat or antimouse secondary antibodies conjugated with Alexa Fluor $488\left(2 \mu \mathrm{gL}^{-1}\right.$ for 45 min; Jackson ImmunoResearch, West Grove, PA, U.S.A.). Cell nuclei were stained with SYTOX Orange (1 : 5000 dilution for $20 \mathrm{~min}$, Molecular Probes, Eugene, OR, U.S.A.). Sections were mounted in ProLong Gold (Molecular Probes) for $24 \mathrm{~h}$. Sections were scanned on a Leica SP8 microscope equipped with a Coherent Chameleon MP laser. Excitation of Alexa Fluor 488 was elicited using two-photon excitation at 


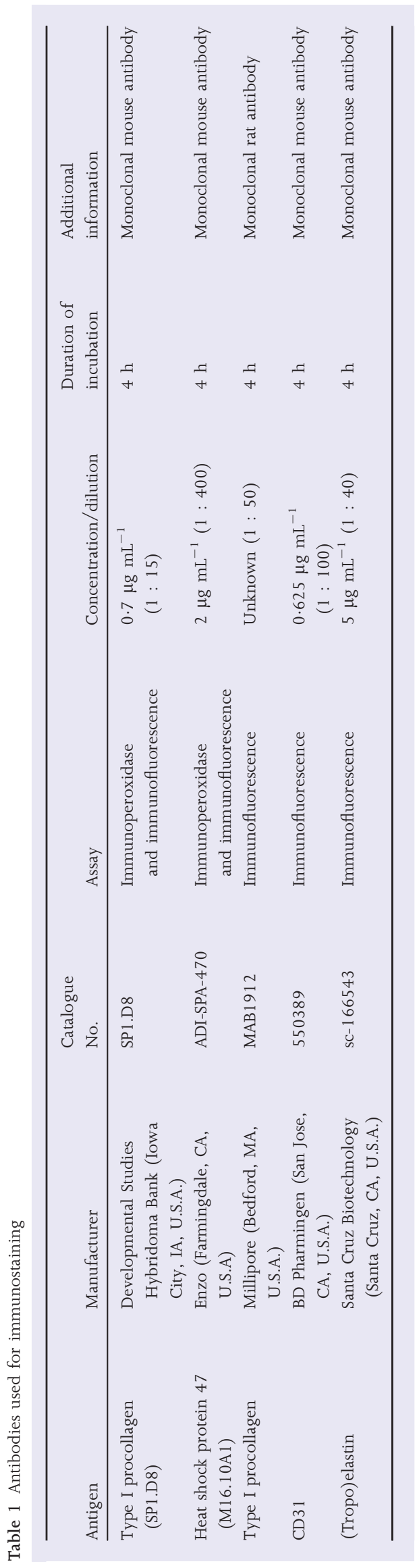

$775 \mathrm{~nm}$. Fluorescent emissions were detected by a HyD detector through an acousto-optical multibeam splitter between 490-535 nm (Alexa Fluor 488) and 565-605 nm (SYTOX Orange). SHG emissions were elicited using two-photon excitation at $820 \mathrm{~nm}$ and detected through an externally mounted narrow 410/10 bandwidth filter. Forward emissions were detected by an externally mounted forward-directed photomultiplier tube with gain no greater than $\sim 800 \mathrm{mV}$. For some sections, backward signals were detected using an external Leica HyD detector. Images were captured at 12 bits using $2 \times \max$ spatial resolution determined by Nyquist sampling parameters per objective. Image stacks were analysed using ImageJ v.1.49t (http://imagej.nih.gov/ij/), 3-D Gaussian blurred at $1 \times$ spatial resolution, and projected using maximum projection or $+1 \mathrm{SD}$ projections. Flattened stack images were background subtracted using an average value within an area at least $15 \times 15 \mu \mathrm{m}$ known to contain only background. Paired images were adjusted until an equal percentage of pixels in each image were saturated. To analyse cross-sectional width, 15-30 collagen fibrils were measured per skin sample. Threedimensional renders were generated with FluoRender software (v.2.16, University of Utah, Salt Lake City, UT, U.S.A.). ${ }^{30,31}$

\section{Statistics}

Group differences were assessed using one-way ANOvA tests with Tukey multiple comparisons adjustment. When appropriate, logarithmic transformations were performed to achieve normality. The Welch-Satterthwaite t-test was used to determine significant group differences in postpartum skin samples. All statistical tests were two-sided using an overall alpha level of 0.05 to determine statistical significance. Data were analysed using SAS software v.9.3 (SAS Institute Inc., Cary, NC, U.S.A.).

\section{Results}

\section{Participants}

Participants included pregnant women (28 participants; 22 white women and six black or biracial women; mean age $24.9 \pm 1.0$ years) who had recently developed erythematous abdominal SG (average time of onset 27.4 $\pm 1 \cdot 2$ weeks of pregnancy; mean duration $6.0 \pm 1.0$ weeks at the time of skin sampling). Overall, 19 participants had a family history of SG, 11 had past pregnancies, and six had SG from previous pregnancies. For each participant, we obtained skin samples from: (i) the centre of SG lesions; (ii) perilesional, normal-appearing, stretched abdominal skin located 2-3 cm from SG lesions; and (iii) minimally stretched normal-appearing skin from the hip (control). ${ }^{4}$

\section{Marked separation of collagen bundles in early striae gravidarum}

In order to investigate the organization of collagen bundles in early SG, we used multiphoton microscopy to perform SHG imaging, which provides high-resolution, three-dimensional 


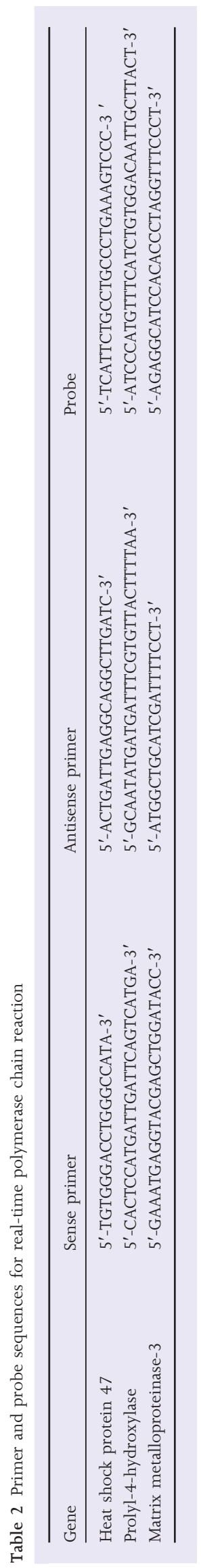

imaging of fibrillar collagen based on its inherent optical properties (i.e. exogenous staining or labelling of collagen is not required). ${ }^{32}$ As type I collagen is the most abundant fibrillar collagen in the dermal ECM of human skin, ${ }^{8,10}$ SHG imaging predominantly reflects the structure/organization of this type of collagen. ${ }^{33}$

In control skin from the hip and perilesional abdominal skin, dermal collagen bundles appeared normal, and were organized in a tightly packed, interwoven pattern, especially in the reticular dermis, and to a lesser degree, in the papillary dermis (Fig. 1a, b). In the dermis of SG, there were patchyto-broad areas in which collagen bundles appeared abnormally separated (Fig. 1c). This separation was striking in areas of the reticular dermis, and more modest in the papillary dermis.

\section{Disorganized collagen fibrils lacking organization as bundles in early striae gravidarum}

Given the marked separation of collagen bundles in the reticular dermis of SG, we next performed high-magnification imaging of dermal areas between collagen bundles. In control (data not shown) and perilesional abdominal skin (Fig. 2a), SHG imaging showed narrow spaces between collagen bundles in the reticular dermis. Within many of these slender spaces, immunofluorescence staining of tropoelastin revealed the presence of normal elastic fibres, which were mostly horizontally oriented with respect to the overlying epidermis.

Within the prominent spaces separating collagen bundles in the reticular dermis of SG, high-magnification SHG imaging revealed groups or arrays of loosely packed, wavy collagen fibrils that lacked organization as bundles (Fig. 2b). Mean cross-sectional width of these fibrils was $413 \pm 10 \mathrm{~nm}$ (six subjects). Similar loosely packed, disorganized collagen fibrils were not observed in control or perilesional skin. Three-dimensional video rendering of images clearly demonstrates that the normal organization of collagen bundles in early SG is severely disrupted, with marked separation of bundles and emergence of disorganized, wavy collagen fibrils (Video S1; see Supporting Information).

Additionally, immunofluorescence staining of tropoelastin revealed a dramatic decrease of normal elastic fibres, and in their place, there were numerous disorganized tropoelastinrich 'fibrils' (Fig. 2b). As previously reported, gene expression of tropoelastin was increased, suggesting that these fibrils are newly synthesized. ${ }^{4}$ Many of these tropoelastin-rich fibrils were intermixed with the disorganized collagen fibrils, and some decorated nearby collagen bundles. These observations indicate that disorganized collagen fibrils, in addition to disorganized tropoelastin-rich fibrils, are present within the large spaces separating collagen bundles in SG.

\section{Close proximity of procollagen-producing fibroblasts to disorganized collagen fibrils in early striae gravidarum}

Next, we examined immunofluorescence staining of type I procollagen, the precursor of type I collagen. In the reticular dermis of control (Fig. 3a) and perilesional skin (data not shown), type I procollagen staining occurred in fibroblasts that were 
Fig 1. Marked separation of dermal collagen bundles in early striae gravidarum. To examine collagen bundles, second-harmonic generation imaging of skin sections $(10 \mu \mathrm{m}$ thick) was performed using multiphoton microscopy. Collagen bundles appear white. (a) Minimally stretched, normal-appearing skin from the hip (control) and (b) perilesional, normal-appearing, stretched skin from the abdomen, with both displaying interwoven, tightly packed collagen bundles, especially in the mid-to-deep dermis. Prominent dark areas correspond to the location of dermal blood vessels. (c) Striae gravidarum lesions, with collagen bundles appearing separated and loosely packed, particularly in the mid-to-deep dermis. Bottom panels magnify representative areas of the mid-to-deep dermis. Dotted lines indicate the dermoepidermal junction. Images are representative of 17 participants. Original magnification $\times 20$

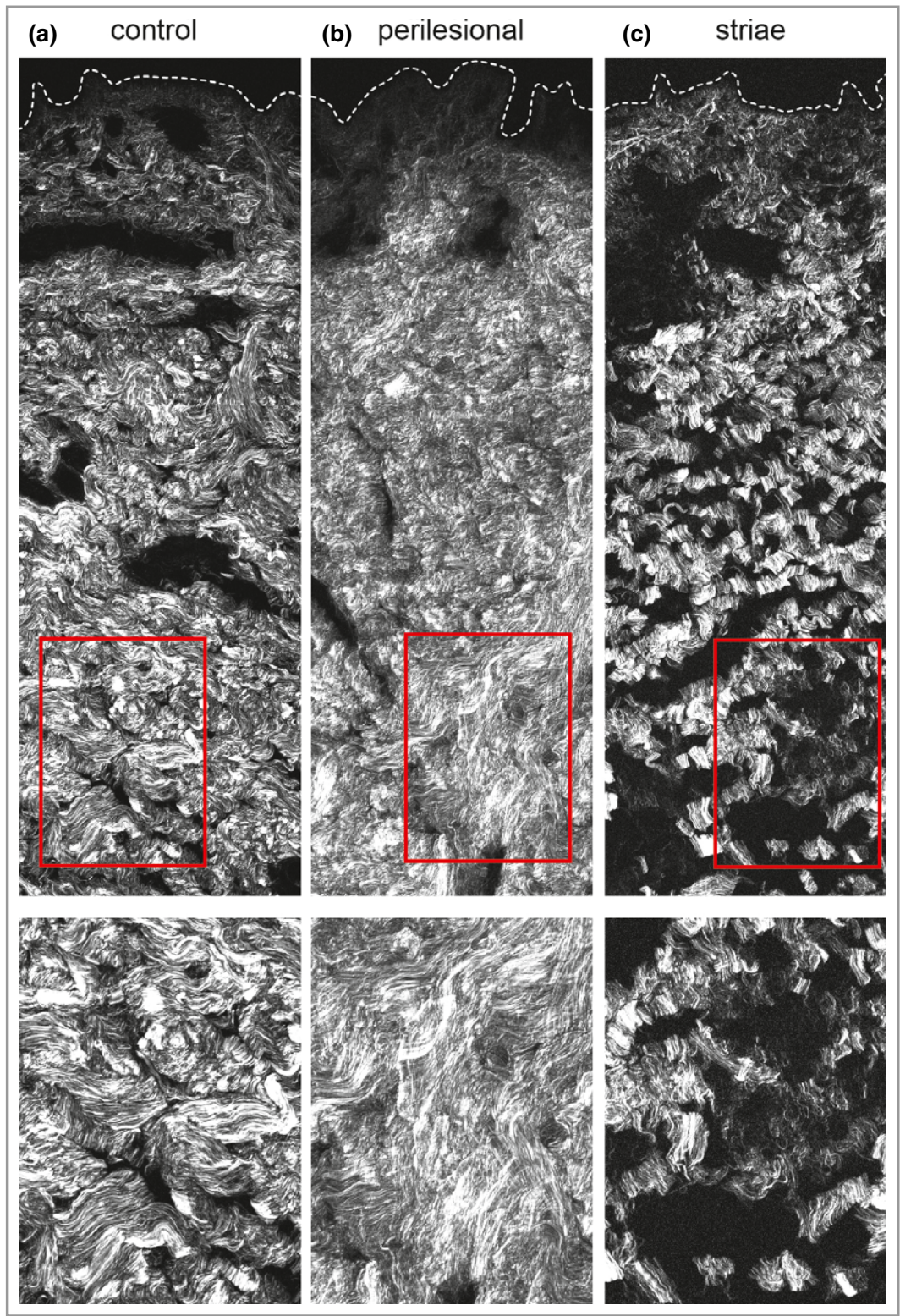

embedded within the interior of collagen bundles or attached to their exteriors. In the reticular dermis of SG, procollagen staining occurred within numerous large fibroblasts (Fig. 3b). These fibroblasts were often localized to the exterior of separated collagen bundles, and in close proximity to the disorganized collagen fibrils within the spaces between bundles. Additionally, some fibroblasts were enmeshed within the disorganized collagen fibrils. The spatial relationship of these procollagen-producing fibroblasts to the disorganized collagen fibrils is clearly illustrated in a three-dimensional video rendering of images (Video S2; see Supporting Information).

\section{Increased type I procollagen expression throughout the dermis in early striae gravidarum}

Having observed type I procollagen expression in large fibroblasts near disorganized collagen fibrils in SG, we next examined immunostaining of type I procollagen throughout the dermis (from superficial to deep dermis). In control or perilesional skin, procollagen staining of fibroblasts was modest throughout the dermis. In SG, procollagen staining was greater, with numerous, large, intensely stained fibroblasts (Fig. 4a). Based on image analysis, the amount of dermal staining was significantly increased $14 \cdot 3 \pm 5 \cdot 7$-fold in SG $(P<0.05)$, compared with control skin. Similarly, SG displayed significantly increased expression of heat shock protein 47, an intracellular chaperone protein required for synthesis of type I procollagen (30.0 $\pm 8 \cdot 2$-fold, $\mathrm{P}<0 \cdot 05$; Fig. 4b).

Consistent with these immunostaining results, gene expression of type I procollagen and heat shock protein 47 was significantly elevated $7 \cdot 3 \pm 3 \cdot 4$-fold and $2 \cdot 8 \pm 0 \cdot 7$-fold, respectively, in SG lesions $(\mathrm{P}<0.05$, Fig. 4c) compared with control skin. SG also displayed significantly increased gene expression of prolyl-4-hydroxylase, an enzyme required for 


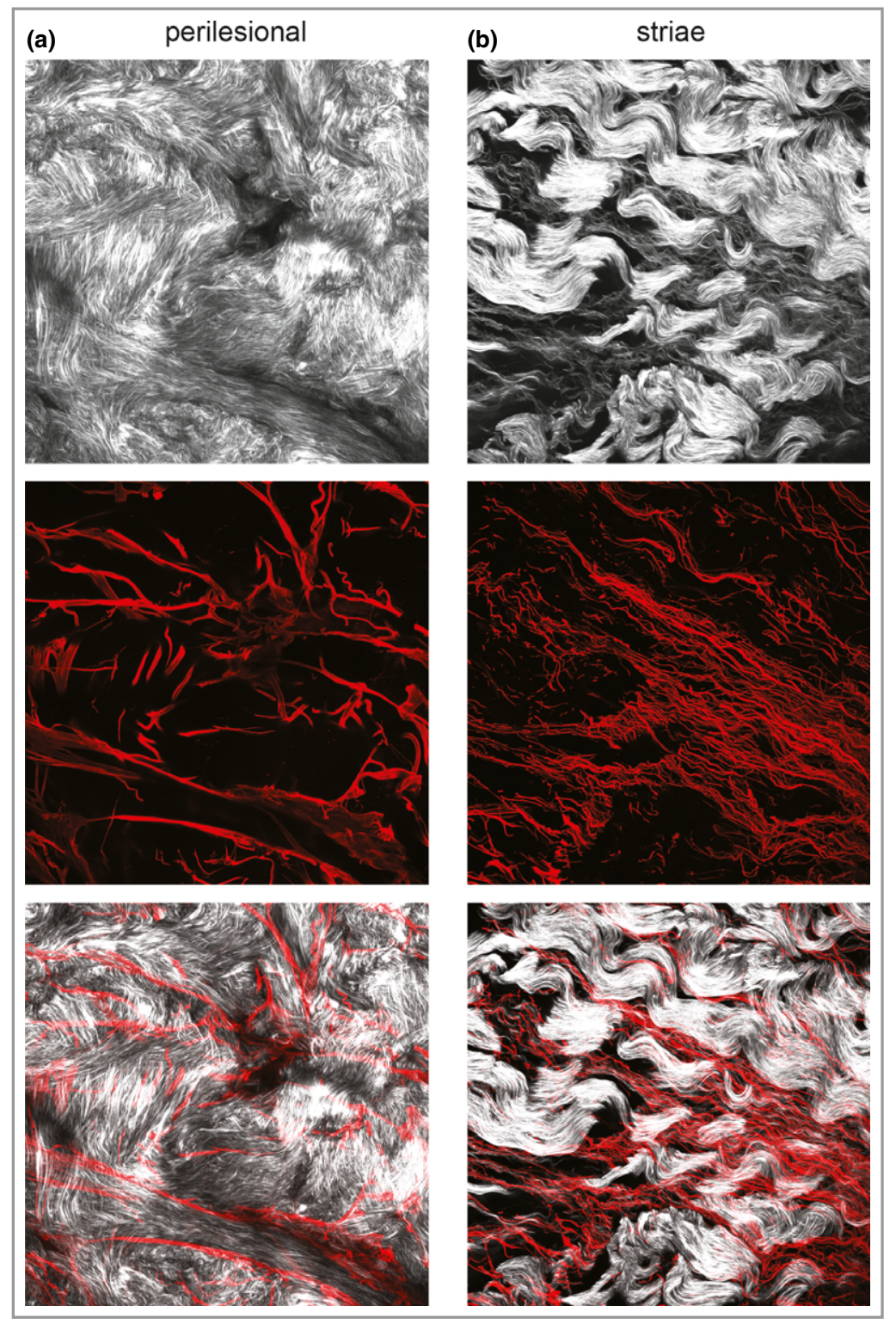

Fig 2. Disorganized collagen fibrils lacking organization as bundles within prominent spaces separating collagen bundles in early striae gravidarum. High-magnification secondharmonic generation imaging and immunofluorescence staining of skin sections (40 $\mu \mathrm{m}$ thick) were performed. Upper panels display collagen fibrils, which appear white. In the middle panels, red represents immunofluorescence staining of tropoelastin, the main component of elastic fibres. Bottom panels show merged images. (a) Mid-to-deep dermis of perilesional abdominal skin, displaying collagen fibrils organized as densely packed, interwoven bundles separated by narrow spaces (top panel). Intact elastic fibres were present (middle panel) and mostly localized to the slender spaces between collagen bundles (bottom panel). (b) Mid-todeep dermis of striae gravidarum, displaying collagen bundles separated by prominent spaces containing arrays of disorganized, wavy collagen fibrils (top panel). Numerous disorganized tropoelastin-rich fibrils were present in the prominent spaces (middle panel), and intermixed with disorganized collagen fibrils (bottom panel). Second harmonic generation images are more completely shown in three-dimensional video rendering (Video S1; see Supporting Information). Images are representative of 13 participants. Original magnification $\times 40$.

stable assembly of type I collagen fibrils $(3 \cdot 0 \pm 0 \cdot 8$-fold, $P \leq 0.05$ compared with control skin; Fig. 4c), and increased expression of collagen-stimulating growth factors, including transforming growth factor- $\beta 1$ and $-\beta 3$ and connective tissue growth factor $(\mathrm{P}<0.05$ compared with control skin and/or perilesional skin; Fig. 4c). Finally, we found no significant changes in gene expression of enzymes that fragment type I collagen fibrils, including matrix metalloproteinase-1 and -3 (data not shown). These data indicate that synthesis, but not enzymatic fragmentation, of type I collagen is increased in early SG.

\section{Prominent vasculature in early striae gravidarum}

As early SG are erythematous and the organization of the collagenous ECM can influence the formation and organization of dermal blood vessels, ${ }^{34}$ we investigated vascular endothelial cells by CD31 immunofluorescence staining. In control (Fig. 5a) and perilesional skin (data not shown), vascular endothelial cells formed normal-appearing, discrete, singular, tubular vessels in the papillary and reticular dermis. In the papillary and reticular dermis of SG, CD31 staining revealed collections of numerous, branching, widened, intertwined vessels, which tended to localize to the prominent spaces formed by separation of collagen bundles (Fig. 5b). Video S3 (see Supporting Information) shows this complex, lobule-like organization of vessels in a three-dimensional video rendering. These data reveal that there is abnormally increased prominence and complexity of vasculature in early SG.

\section{Persistence of disorganized collagen fibrils and increased type I procollagen expression in postpartum striae gravidarum}

Finally, we investigated collagen organization and production in postpartum women with SG. For each participant, we obtained biopsies of erythematous, abdominal SG and 
Fig 3. Close proximity of procollagenproducing fibroblasts to disorganized collagen fibrils in early striae gravidarum. Highmagnification second-harmonic generation imaging and immunofluorescence staining of skin sections (30 $\mu \mathrm{m}$ thick) were performed. Upper panels display collagen fibrils, which appear white. In the middle panels, blue represents cell nuclei, and red represents immunofluorescence staining of type I procollagen. Bottom panels show merged images. (a) Mid-to-deep dermis of control skin from the hip, displaying densely packed, interwoven collagen bundles (top panel). Type I procollagen staining appeared within fibroblasts (middle panel) that were mostly embedded within the interior of collagen bundles or attached to their exteriors (bottom panel). (b) Mid-to-deep dermis of striae gravidarum, displaying collagen bundles separated by prominent spaces containing groups of disorganized, wavy collagen fibrils (top panel). Type I procollagen staining appeared within numerous large fibroblasts (middle panel) that were mostly localized to the exterior of separated collagen bundles, in close proximity to the disorganized collagen fibrils (bottom panel). Merged images are more completely shown in three-dimensional video rendering (Video S2; see Supporting Information). Images are representative of four participants. Original magnification $\times 40$.
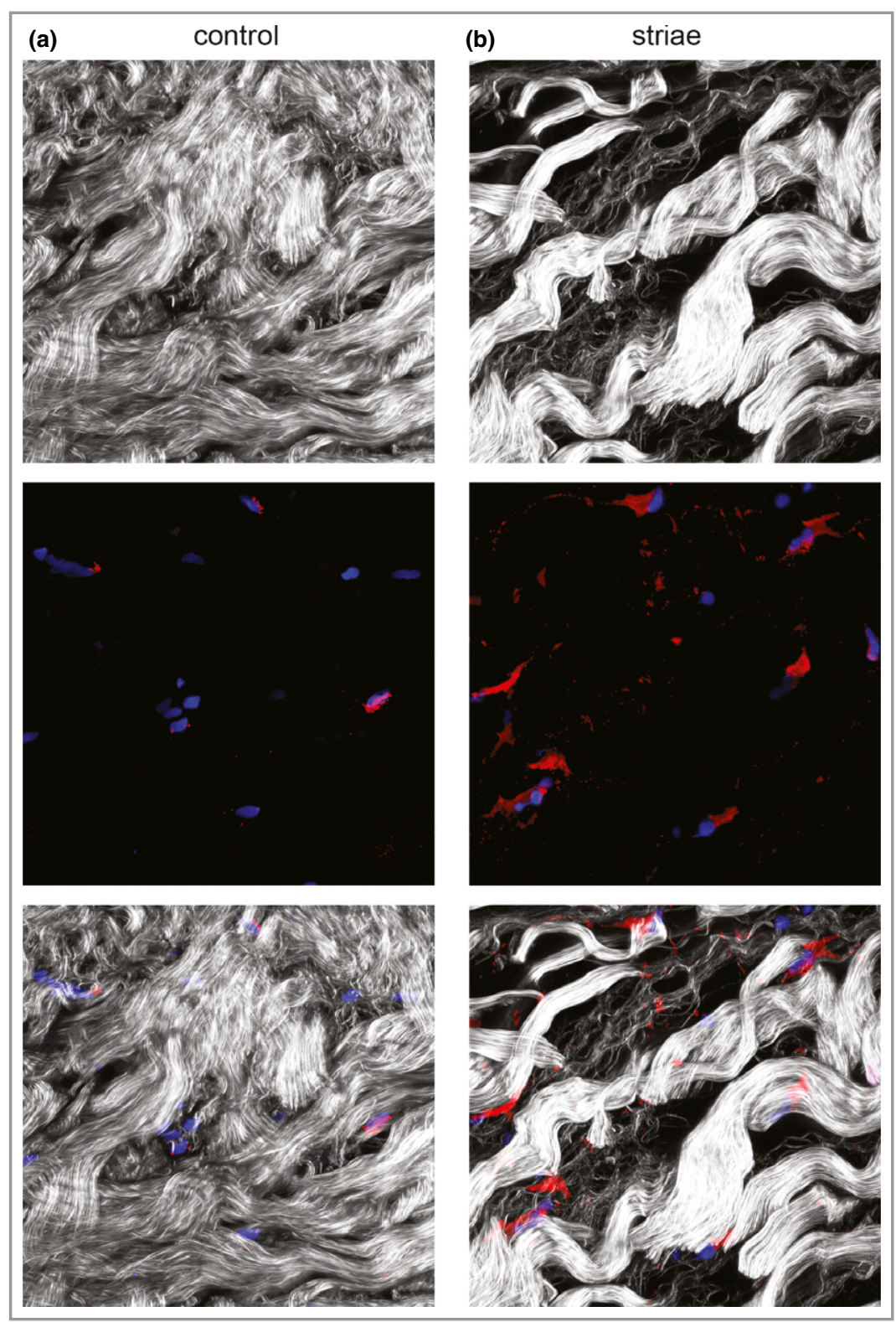

perilesional skin at 4-8 weeks postpartum. These biopsies came from a subset of the same participants that had biopsies of early SG taken during pregnancy (nine participants; seven white and two black or biracial women). ${ }^{4}$

In postpartum perilesional skin, we found that the organization of dermal collagen bundles and type I procollagen staining appeared similar to control and perilesional skin during pregnancy (Fig. 6a). Importantly, postpartum SG continued to display patchy-to-broad areas with marked separation of collagen bundles, especially the reticular dermis (Fig. 6b). Between separated bundles, there remained numerous groups of wavy, disorganized collagen fibrils that were similar in appearance and thickness to those observed in SG during pregnancy (mean cross-sectional width $431 \pm 9 \mathrm{~nm}$, five subjects). Disorganized tropoelastin-rich fibrils also remained present in these areas (data not shown), consistent with previous findings. ${ }^{4}$ There were numerous, large type I procollagen-positive fibroblasts in close proximity to the disorganized collagen fibrils (Fig. 6b). In addition, type I procollagen gene expression remained significantly elevated $9 \cdot 5 \pm 2 \cdot 6$-fold in postpartum SG, compared with postpartum perilesional skin $(P<0.05$, Fig. 6c). Overall, these data demonstrate that abnormalities in the structure, organization and production of collagen fibrils persist into the postpartum period.

\section{Discussion}

Despite the prevalence of SG, a comprehensive analysis of collagen fibrils in early lesions has been lacking. Here, we observed that early SG displayed severely disrupted organization of dermal collagen fibrils, with marked separation of bundles, especially in patchy-to-extensive areas of the mid-to-deep dermis. 


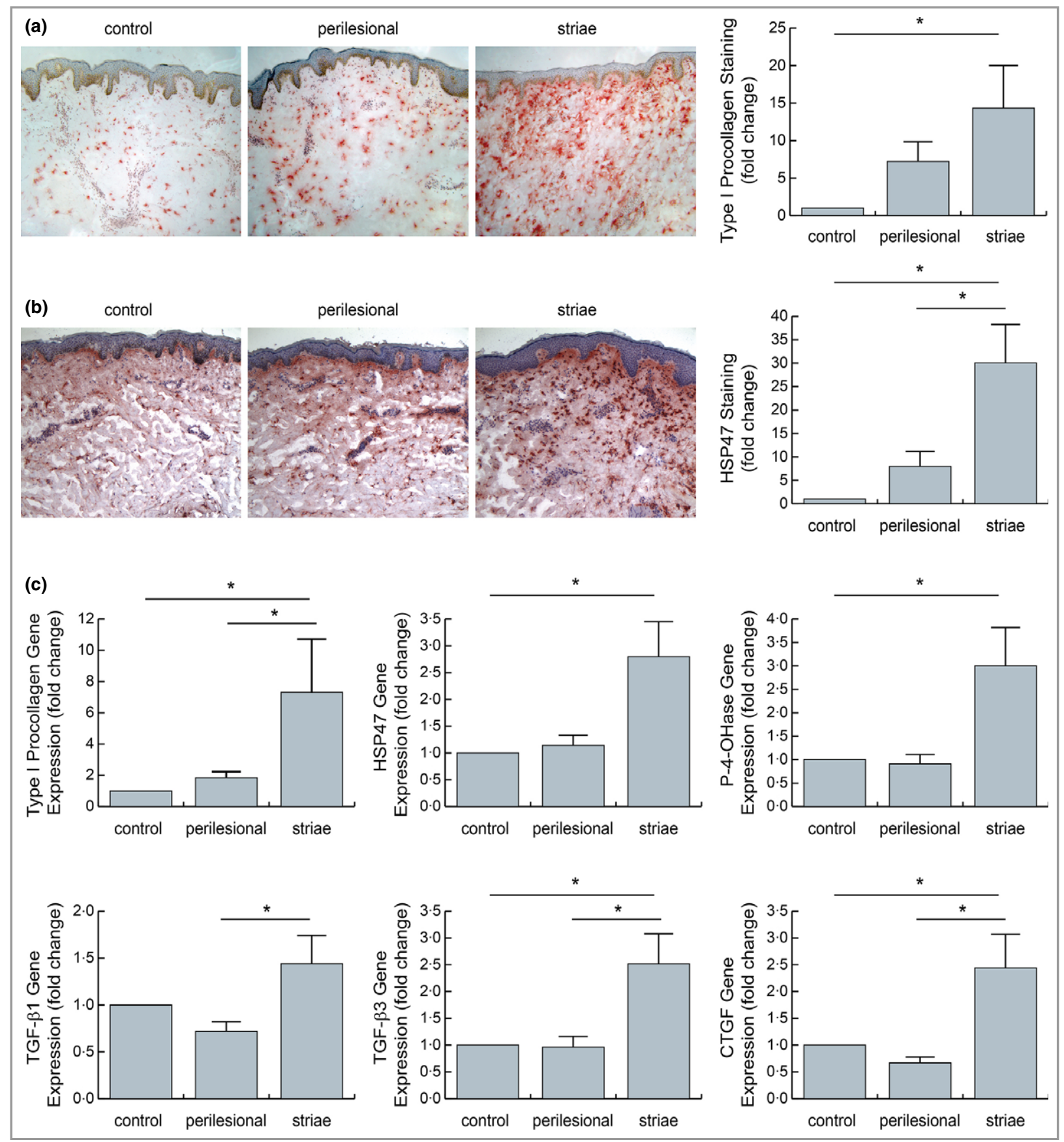

Fig 4. Increased expression of type I procollagen in fibroblasts throughout the dermis in early striae gravidarum. Immunostaining of skin sections (7 $\mu$ m thick) for (a) type I procollagen $(n=11)$ and (b) heat shock protein (HSP) $47(n=11)$, a chaperone protein required for type I procollagen synthesis. Original magnification $\times 10$. Bar graphs show quantification of staining by image analysis. (c) Using real-time polymerase chain reaction, gene expression of type I procollagen $(n=17)$, HSP47 $(n=12)$, and prolyl-4-hydroxylase $(n=11$, an enzyme required for the stable assembly of type I collagen fibrils) was assessed in skin samples. Gene expression of collagen-stimulating growth factors, including transforming growth factor (TGF)- $\beta 1(\mathrm{n}=10)$, TGF- $\beta 3(\mathrm{n}=11)$ and connective tissue growth factor (CTGF) $(\mathrm{n}=11)$, was also measured. All bar graphs show mean fold change $\pm \mathrm{SE} . * \mathrm{P} \leq 0 \cdot 05$.

We considered that enzyme-mediated fragmentation of collagen fibrils may promote these alterations, but did not observe increased expression of matrix metalloproteinase- 1 or -3 , which are the predominant enzymes that fragment dermal type I collagen fibrils. As previously reported, ${ }^{4}$ early SG did not display increased inflammatory cells, including neutrophils, mast cells or macrophages, which produce enzymes that fragment collagen and other ECM components. Although we cannot rule out that increased enzymatic or cellular fragmentation of collagen fibrils occurs before lesions become 
Fig 5. Prominent dermal vasculature in early striae gravidarum. High-magnification secondharmonic generation imaging and immunofluorescence staining of skin sections (60 $\mu \mathrm{m}$ thick) were performed. Upper panels display collagen fibrils, which appear white. In the middle panels, $\mathrm{CD} 31$

immunofluorescence staining of vascular endothelial cells appears green. Bottom panels show merged images. (a) In the mid-to-deep dermis of control skin, collagen bundles were densely packed (top panel), and CD31 staining localized to thin, tube-like vessels (middle panel), which were situated between collagen bundles (bottom panel). (b) In the mid-to-deep dermis of striae gravidarum, collagen bundles appeared markedly separated (top panel), and CD31 staining appeared increased, highlighting the presence of multiple, branching, widened, intertwined, tube-like vessels (middle panel), which were localized to the spaces separating collagen bundles (bottom panel). Merged images are more completely shown in three-dimensional video rendering (Video S3; see Supporting Information). Images are representative of four participants. Original magnification $\times 40$.
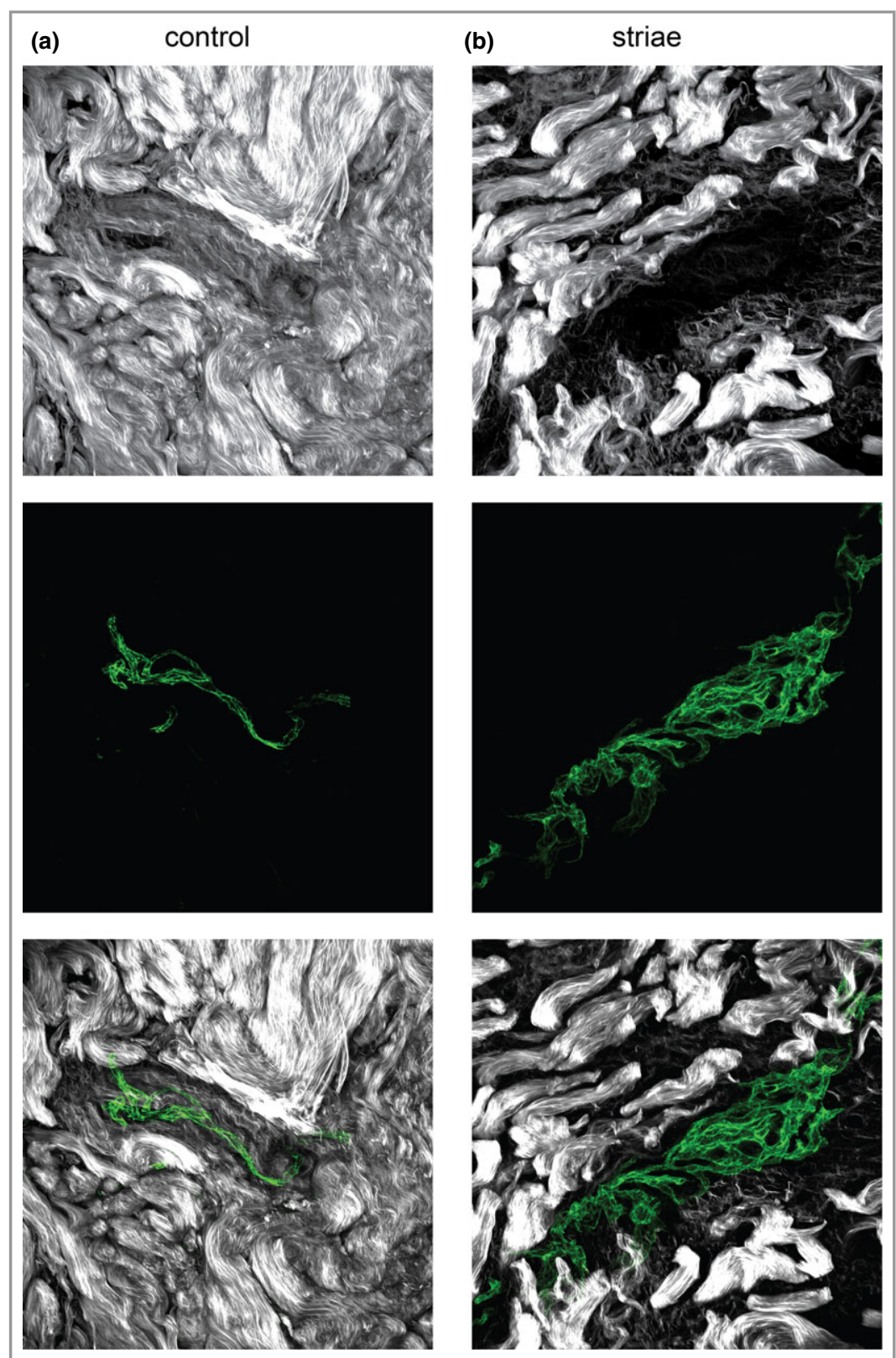

clinically apparent, this possibility is unlikely, as we investigated early SG that were still evolving (i.e. in the process of developing and maturing).

Therefore, intense skin stretching that mechanically 'pulls apart' the dermal ECM may disrupt the organization of collagen bundles. ${ }^{4,35}$ This hypothesis suggests that, in predisposed individuals, the dermal ECM lacks the ability to accommodate intense skin stretching. ${ }^{36}$ Such a hypothesis might explain why SG often occur on the abdomen, which experiences the greatest stretching of any area of skin during pregnancy. While this hypothesis requires further investigation, a recent study suggests that skin stretching plays a role in SG development, as maternal baseline weight, weight gain during pregnancy and multiple gestational pregnancy appear to be risk factors. ${ }^{37}$ Additional support comes from histological and clinical observations of skin stretching produced by surgical tissue expanders, which can promote separation of dermal collagen bundles $^{38}$ and, occasionally, development of striae within 1-3 months. ${ }^{39}$

In the spaces between separated collagen bundles in SG, we found groups of disorganized, wavy collagen fibrils, in addition to numerous disorganized tropoelastin-rich fibrils. ${ }^{4}$ While some of these collagen fibrils may arise as a consequence of damage to pre-existing collagen bundles, our data suggest that they mainly result from de novo synthesis by dermal fibroblasts. Indeed, numerous large type I procollagen-expressing fibroblasts were observed to be in close proximity to the fibrils. Additionally, staining of procollagen-expressing fibroblasts was increased throughout the dermis of SG. The upregulation of type I procollagen production and synthesis of disorganized 

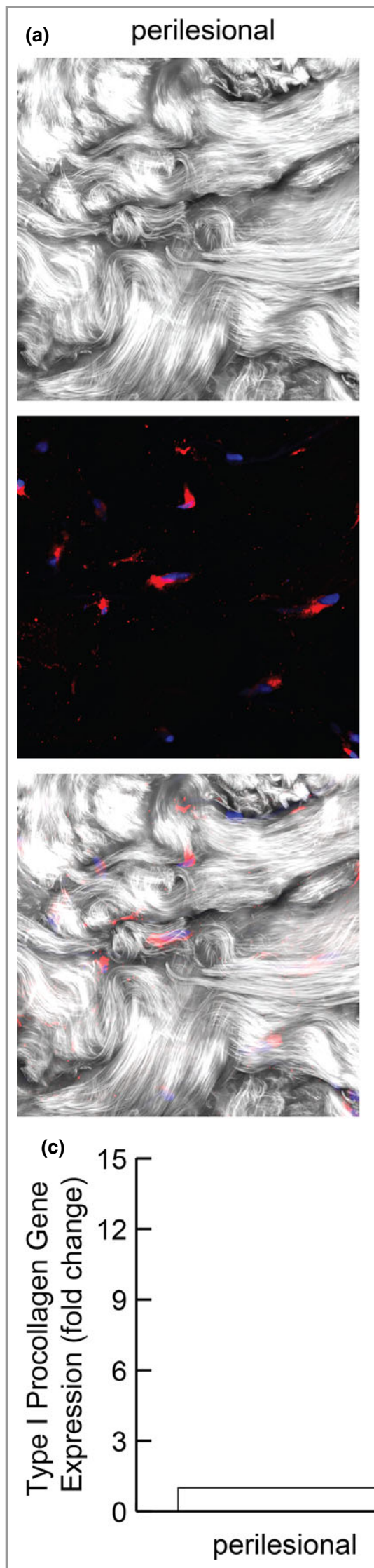

(b)
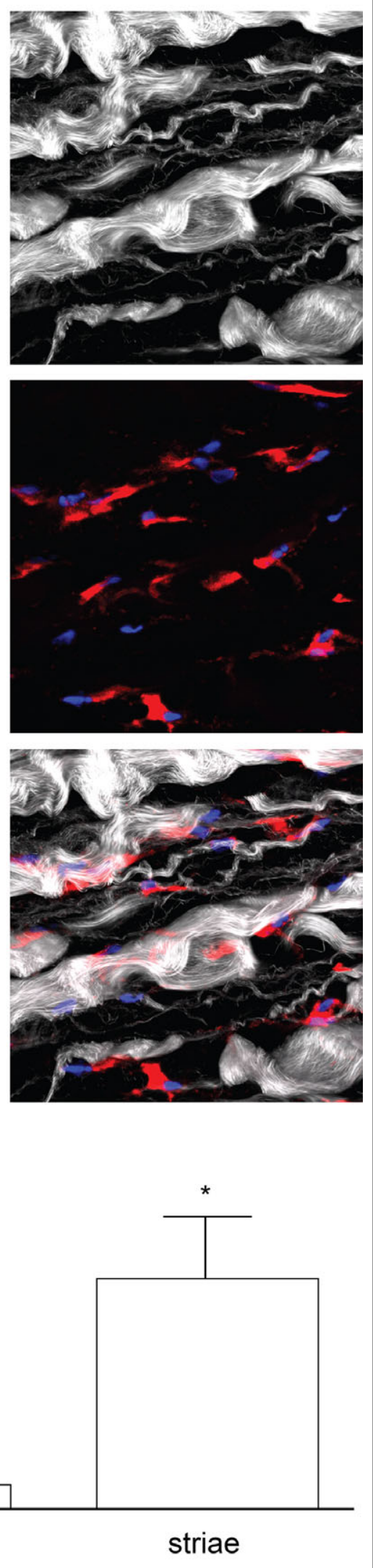

Fig 6. Persistence of disorganized collagen fibrils and elevated type I procollagen expression in postpartum striae gravidarum. At 4-8 weeks postpartum, skin samples of recently developed abdominal striae gravidarum and normal-appearing perilesional abdominal skin were obtained from a subset of the same participants who had biopsies taken during pregnancy. High-magnification second-harmonic generation imaging and immunofluorescence staining of skin sections (100 $\mu \mathrm{m}$ thick) were performed. Upper panels display collagen fibrils, which appear white. In the middle panels, cell nuclei appear blue, and immunofluorescence staining of type I procollagen appears red. Bottom panels show merged images. (a) Mid-to-deep dermis of perilesional skin, in which collagen bundles continued to appear interwoven and densely packed (top panel). Type I procollagen staining continued to localize to fibroblasts (middle panel) that were embedded within the interior of collagen bundles or attached to their exteriors (bottom panel). (b) Mid-to-deep dermis of postpartum striae gravidarum, in which collagen bundles continued to appear separated by prominent spaces containing numerous disorganized, wavy collagen fibrils (top panel). Many large fibroblasts staining for type I procollagen remained present (middle panel), and were localized to the exterior of collagen bundles, in close proximity to disorganized collagen fibrils (bottom panel). Images are representative of five participants. Original magnification $\times 40$. (c) Gene expression of type I procollagen $(n=7)$ was assessed in skin samples (mean fold change $\pm \mathrm{SE}$ ). $* \mathrm{P}<0.05$. 
collagen fibrils, which apparently last for at least 6-8 weeks postpartum, may represent a reparative response to disruption or 'wounding' of normal collagen bundles, a response to intense skin stretching, ${ }^{8,40}$ or a combination of both.

Interestingly, disorganized collagen fibrils persisted into the early postpartum period and failed to form normal, densely packed, interwoven collagen bundles. We considered that collagen bundles may form with greater time, but we found that loosely packed, disorganized fibrils remain present even in mature, hypopigmented SG (data not shown). Bundles of collagen fibrils provide strength and support to human skin. Therefore, separation or damage of collagen bundles, coupled with suboptimal repair producing disorganized fibrils, likely translates to decreased dermal structural support. Loss of structural support precedes and, ultimately, may manifest clinically as dermal atrophy in mature SG. These changes occur despite substantial stimulation of the collagen-producing pathway.

The reasons that disorganized collagen fibrils persist in SG are not clear. Elastic fibres are disrupted and tropoelastin-rich fibrils in SG are unable to organize into normal-appearing elastic fibres, possibly because lesions lack ECM components essential for proper elastic fibre assembly. ${ }^{4}$ Similarly, ECM components involved in organizing collagen fibrils into bundles may be deficient or dysfunctional in early SG. Such organizational components may include elastic fibres, crosslinking enzymes (e.g. lysyl oxidase), other types of collagen (e.g. type $\mathrm{V}$ and XI collagen), proteoglycans (e.g. decorin, biglycan, lumican), glycoproteins (e.g. fibronectin) and glycosaminoglycans (e.g. hyaluronic acid, dermatan sulfate)., 10,13-17,40,41 For example, small leucine-rich proteoglycans, such as decorin and biglycan, may play a role in regulating collagen fibril assembly, diameter, length and organization. ${ }^{16,42}$ Additionally, increased mechanical tension of skin during pregnancy may play a role in preventing the formation of collagen bundles, a situation possibly analogous to impaired healing or 'spreading' of surgical wounds under high mechanical tension. ${ }^{43}$ Finally, it is possible that hormonal changes during pregnancy may contribute to persistent disorganization of collagen fibrils in SG. ${ }^{40,44}$ Additional research may support these possibilities.

In addition to changes in collagen fibrils, we observed increased prominence of dermal blood vessels in early SG. This change, involving increased number, branching and widening of vessels, likely promotes clinical erythema and explains how the appearance of striae can be improved, in part, by laser therapies targeting dermal vasculature. ${ }^{45}$ Interestingly, fragmentation of type I collagen fibrils, and hence decreased density of the collagenous ECM, can induce endothelial cells to form vascular tubes. ${ }^{34}$ Similarly, in early SG, dispersal of collagen bundles may promote increased prominence or formation of blood vessels. Dispersal of collagen bundles may also promote clinical erythema by allowing easier visualization of colour derived from deep dermal vessels. We considered that inflammation may contribute to erythema, but as previously reported, ${ }^{4}$ infiltrating immune cells are not substantially present in early SG.
Taken together, our data suggest that strategies aimed at promoting organization of collagen fibrils into densely packed bundles that provide structural support may be effective for preventing or treating SG. In this regard, potential therapies may include topical retinoids, which can improve the clinical appearance of early striae lesions. ${ }^{46}$ Some limitations of this type of therapy include the fact that topical retinoids are typically avoided during pregnancy, and it remains unclear exactly how these agents impact collagen bundling or whether they can prevent new lesion development.

Finally, it is interesting to note that disruption coupled with suboptimal repair is observed for both elastic fibres ${ }^{4}$ and collagen bundles in SG. Furthermore, abnormal collagen fibrils and tropoelastin-rich fibrils localize together in the spaces created by disruption of the normal dense architecture of the collagenous dermal ECM. These observations suggest that the organization of collagen bundles and elastic fibres may be interdependent. ${ }^{40}$ Investigating the potential role of this interdependence may provide important insights into the pathophysiology of SG and the fundamental regulation (or biogenesis) of the human dermal ECM.

\section{Acknowledgments}

Heather Chubb MS provided statistical analyses, Jennifer Bell CCRP coordinated subject recruitment, and Laura VanGoor BFA provided assistance with graphical material. All are employees of the Department of Dermatology at the University of Michigan Medical School. Pamela Sharaf, an undergraduate student at the University of Michigan, provided technical assistance with the laboratory assays.

\section{References}

1 Chang AL, Agredano YZ, Kimball AB. Risk factors associated with striae gravidarum. J Am Acad Dermatol 2004; 51:881-5.

2 Salter SA, Kimball AB. Striae gravidarum. Clin Dermatol 2006; 24:97-100.

3 Korgavkar K, Wang F. Stretch marks during pregnancy: a review of topical prevention. Br J Dermatol 2015; 172:606-15.

4 Wang F, Calderone K, Smith NR et al. Marked disruption and aberrant regulation of elastic fibres in early striae gravidarum. Br J Dermatol 2015; 173:1420-30.

5 Langton AK, Graham HK, McConnell JC et al. Organisation of the dermal matrix impacts the biomechanical properties of skin. Br J Dermatol 2017; 177:818-27.

6 Kielty CM, Sherratt MJ, Shuttleworth CA. Elastic fibres. J Cell Sci 2002; 115:2817-28.

7 Lewis KG, Bercovitch L, Dill SW, Robinson-Bostom L. Acquired disorders of elastic tissue: part I. Increased elastic tissue and solar elastotic syndromes. J Am Acad Dermatol 2004; 51:1-21; quiz 2-4.

8 Silver FH, Siperko LM, Seehra GP. Mechanobiology of force transduction in dermal tissue. Skin Res Technol 2003; 9:3-23.

9 Fang M, Goldstein EL, Turner AS et al. Type I collagen D-spacing in fibril bundles of dermis, tendon, and bone: bridging between nano- and micro-level tissue hierarchy. ACS Nano 2012; 6:950314.

10 Fisher GJ, Varani J, Voorhees JJ. Looking older: fibroblast collapse and therapeutic implications. Arch Dermatol 2008; 144:666-72. 
11 Shoulders MD, Raines RT. Collagen structure and stability. Annu Rev Biochem 2009; 78:929-58.

12 van Zuijlen PP, Ruurda JJ, van Veen HA et al. Collagen morphology in human skin and scar tissue: no adaptations in response to mechanical loading at joints. Burns 2003; 29:423-31.

13 Smith LT, Holbrook KA, Madri JA. Collagen types I, III, and V in human embryonic and fetal skin. Am J Anat 1986; 175:507-21.

14 Iozzo RV. Matrix proteoglycans: from molecular design to cellular function. Annu Rev Biochem 1998; 67:609-52.

15 Wenstrup RJ, Florer JB, Brunskill EW et al. Type V collagen controls the initiation of collagen fibril assembly. J Biol Chem 2004; 279:53331-7.

16 Kalamajski S, Oldberg A. The role of small leucine-rich proteoglycans in collagen fibrillogenesis. Matrix Biol 2010; 29:248-53.

17 Kadler KE, Hill A, Canty-Laird EG. Collagen fibrillogenesis: fibronectin, integrins, and minor collagens as organizers and nucleators. Curr Opin Cell Biol 2008; 20:495-501.

18 Pinkus H, Keech MK, Mehregan AH. Histopathology of striae distensae, with special reference to striae and wound healing in the Marfan syndrome. J Invest Dermatol 1966; 46:283-92.

19 Shuster S. The cause of striae distensae. Acta Derm Venereol Suppl (Stockh) 1979; 59:161-9.

20 Mitts TF, Jimenez F, Hinek A. Skin biopsy analysis reveals predisposition to stretch mark formation. Aesthet Surg J 2005; 25:593600 .

21 Epstein NN, Epstein WL, Epstein JH. Atrophic striae in patients with inguinal intertrigo: pathogenesis. Arch Dermatol 1963; 87: 450-7.

22 Sheu HM, Yu HS, Chang CH. Mast cell degranulation and elastolysis in the early stage of striae distensae. J Cutan Pathol 1991; 18:410-16.

23 Tsuji T, Sawabe M. Elastic fibers in striae distensae. J Cutan Pathol $1988 ; 15: 215-22$.

24 Watson RE, Parry EJ, Humphries JD et al. Fibrillin microfibrils are reduced in skin exhibiting striae distensae. Br J Dermatol 1998; 138:931-7.

25 Kang S, Cho S, Chung JH et al. Inflammation and extracellular matrix degradation mediated by activated transcription factors nuclear factor-kappaB and activator protein-1 in inflammatory acne lesions in vivo. Am J Pathol 2005; 166:1691-9.

26 Quan T, He T, Kang S et al. Solar ultraviolet irradiation reduces collagen in photoaged human skin by blocking transforming growth factor-beta type II receptor/Smad signaling. Am J Pathol 2004; 165:741-51.

27 Quan T, Shao Y, He T et al. Reduced expression of connective tissue growth factor (CTGF/CCN2) mediates collagen loss in chronologically aged human skin. J Invest Dermatol 2010; 130:415-24.

28 Quan T, He T, Kang S et al. Connective tissue growth factor: expression in human skin in vivo and inhibition by ultraviolet irradiation. J Invest Dermatol 2002; 118:402-8.

29 Quan T, He T, Shao Y et al. Elevated cysteine-rich 61 mediates aberrant collagen homeostasis in chronologically aged and photoaged human skin. Am J Pathol 2006; 169:482-90.

30 Wan Y, Otsuna H, Chien CB, Hansen C. An interactive visualization tool for multi-channel confocal microscopy data in neurobiology research. IEEE Trans Vis Comput Graph 2009; 15:1489-96.

31 Wan Y, Otsuna H, Chien CB, Hansen C. FluoRender: an application of 2D image space methods for $3 \mathrm{D}$ and $4 \mathrm{D}$ confocal microscopy data visualization in neurobiology research. IEEE Pac Vis Symp 2012; 1:201-8.

32 Zipfel WR, Williams RM, Christie R et al. Live tissue intrinsic emission microscopy using multiphoton-excited native fluorescence and second harmonic generation. Proc Natl Acad Sci USA 2003; 100:7075-80.

33 Cox G, Kable E, Jones A et al. 3-dimensional imaging of collagen using second harmonic generation. J Struct Biol 2003; 141:53-62.

34 Varani J, Perone P, Warner RL et al. Vascular tube formation on matrix metalloproteinase-1-damaged collagen. Br J Cancer 2008; 98:1646-52.

35 Arem AJ, Kischer CW. Analysis of striae. Plast Reconstr Surg 1980; 65:22-9.

36 Gilmore SJ, Vaughan BL Jr, Madzvamuse A, Maini PK. A mechanochemical model of striae distensae. Mathl Biosci 2012; 240: $141-7$.

37 Picard D, Sellier S, Houivet E et al. Incidence and risk factors for striae gravidarum. J Am Acad Dermatol 2015; 73:699-700.

38 Verhaegen PD, Schouten HJ, Tigchelaar-Gutter W et al. Adaptation of the dermal collagen structure of human skin and scar tissue in response to stretch: an experimental study. Wound Repair Regen 2012; 20:658-66.

39 Basile FV, Basile AV, Basile AR. Striae distensae after breast augmentation. Aesthetic Plast Surg 2012; 36:894-900.

40 Lapière CM. The ageing dermis: the main cause for the appearance of 'old' skin. Br J Dermatol 1990; 122(Suppl. 35):5-11.

41 Langton AK, Tsoureli-Nikita E, Griffiths CEM et al. Lysyl oxidase activity in human skin is increased by chronic ultraviolet radiation exposure and smoking. Br J Dermatology 2017; 176:1376-8.

42 Reese SP, Underwood CJ, Weiss JA. Effects of decorin proteoglycan on fibrillogenesis, ultrastructure, and mechanics of type I collagen gels. Matrix Biol 2013; 32:414-23.

43 Burgess LP, Morin GV, Rand M et al. Wound healing. Relationship of wound closing tension to scar width in rats. Arch Otolaryngol Head Neck Surgery 1990; 116:798-802.

44 Cordeiro RC, Zecchin KG, de Moraes AM. Expression of estrogen, androgen, and glucocorticoid receptors in recent striae distensae. Int J Dermatol 2010; 49:30-2.

45 Al-Himdani S, Ud-Din S, Gilmore S, Bayat A. Striae distensae: a comprehensive review and evidence-based evaluation of prophylaxis and treatment. Br J Dermatol 2014; 170:527-47.

46 Kang S, Kim KJ, Griffiths CE et al. Topical tretinoin (retinoic acid) improves early stretch marks. Arch Dermatol 1996; 132:519-26.

\section{Supporting Information}

Additional Supporting Information may be found in the online version of this article at the publisher's website:

Video S1 Three-dimensional video rendering of images of collagen bundles in early striae gravidarum.

Video S2 Three-dimensional video rendering of images of synthetically active fibroblasts in early striae gravidarum.

Video S3 Three-dimensional video rendering of images of dermal vasculature in early striae gravidarum. 